\title{
Sarilumab plus methotrexate suppresses circulating biomarkers of bone resorption and synovial damage in patients with rheumatoid arthritis and inadequate response to methotrexate: a biomarker study of MOBILITY
}

Anita Boyapati ${ }^{*}$, Jérôme Msihid ${ }^{2}$, Stefano Fiore ${ }^{3}$, Janet van Adelsberg ${ }^{1}$, Neil M. H. Graham and Jennifer D. Hamilton ${ }^{1}$

\begin{abstract}
Background: Interleukin 6 (IL-6) signaling plays a key role in the pathophysiology of rheumatoid arthritis (RA) and is inhibited by sarilumab, a human monoclonal antibody blocking the IL-6 receptor alpha (IL-6Ra). The effects of sarilumab plus methotrexate (MTX) on serum biomarkers of joint damage and bone resorption were assessed in two independent studies (phase II (part A) and phase III (part B)) of patients with RA with a history of inadequate response to MTX from the MOBILITY study (NCT01061736).

Methods: Serum samples were analyzed at baseline and prespecified posttreatment time points. Biomarkers of tissue destruction, cartilage degradation, and synovial inflammation were measured in part A; assessment of these markers was repeated in part $B$ and included additional analysis of biomarkers of bone formation and resorption (including soluble receptor activator of nuclear factor-kB ligand (sRANKL)). A mixed model for repeated measures was used to compare treatment effects on change in biomarkers. Additionally, changes from baseline in biomarkers were compared between American College of Rheumatology $50 \%$ responders and nonresponders and between patients who achieved or did not achieve low disease activity (LDA), separately by treatment group, at week 24.

Results: In part A, sarilumab 150 and 200 mg every 2 weeks (q2W) significantly reduced biomarkers of tissue destruction, cartilage degradation, and synovial inflammation at both 2 and 12 weeks posttreatment $(p<0.05$ vs placebo). These results were replicated in part B, with markers of these damaging processes reduced at weeks 2 and 24 ( $p<0.05$ vs placebo). Additionally, sarilumab $200 \mathrm{mg}$ q2w significantly reduced both sRANKL and sRANKL/ osteoprotegerin ratio at week 24 ( $p<0.01$ vs placebo). Trends for reduction were noted for several biomarkers in patients who achieved LDA compared with those who did not.

(Continued on next page)
\end{abstract}

\footnotetext{
* Correspondence: anita.boyapati@regeneron.com

${ }^{1}$ Regeneron Pharmaceuticals, Inc., 777 Old Saw Mill River Road, Tarrytown, NY 10591, USA

Full list of author information is available at the end of the article
} 
(Continued from previous page)

Conclusions: Sarilumab plus MTX significantly suppressed biomarkers of bone resorption and joint damage, as compared with placebo plus MTX, in patients with RA. Additional work is needed to determine whether differences in biomarker profiles at baseline or posttreatment can identify patients who achieve improvement in disease activity.

Trial registration: ClinicalTrials.gov, NCT01061736, February 2, 2010.

Keywords: Sarilumab, Rheumatoid arthritis, Biomarker, RANKL/OPG

\section{Background}

Rheumatoid arthritis (RA) is an autoimmune disease characterized by chronic overactivation of the inflammatory system and progressive joint destruction [1]. The localized joint symptoms observed in RA result from persistent synovial inflammation associated with damage to articular cartilage and underlying bone $[1,2]$, which may lead to progressively impaired function and disability [3].

Both innate and adaptive immune processes mediated by cytokine activity play a role in the pathophysiology of RA [4]. For example, the concentration of interleukin 6 (IL-6) is increased in the serum and synovial fluid of patients with RA relative to healthy individuals [5] and correlates with disease activity and joint destruction [4]. Elevation of IL-6 concentrations in joints may facilitate synovial fibroblast activation, and bone resorption and joint damage, through osteoclast formation $[6,7]$. The combination of reduced bone formation and increased bone resorption is a characteristic feature of RA [8].

Studies in cell cultures and mouse models have demonstrated the critical role of IL- 6 in the induction of boneresorptive factors (e.g., receptor activator of nuclear factor-kB ligand (RANKL)) and joint-destructive proteins (e.g., matrix metalloproteinases (MMPs)) from osteoclasts and fibroblast-like synoviocytes (FLS) [6, 9-12]. RANKL, which exists in membrane-bound and soluble forms (sRANKL), binds to RANK to induce osteoclast formation, survival, fusion, and activation [13]. Blockade of the IL-6 receptor (IL-6R) inhibits osteoclast formation in vitro and in vivo [14], and the induction of RANKL observed in a collagen-induced arthritis monkey model is suppressed by treatment with the IL-6R antibody tocilizumab [15].

IL-6R inhibition also blunts RANKL production in FLS from patients with RA [9]. Osteoprotegerin (OPG), a decoy receptor for RANKL, binds both forms of RANKL, preventing activation of RANK and inhibiting osteoclastogenesis [13]. The RANKL/OPG ratio regulates the balance between bone turnover and bone formation, with a higher ratio favoring enhanced bone resorption $[13,16]$. The formation of type I collagen fragments, such as carboxy-terminal collagen crosslinks 1 (CTX-1), another indicator of bone turnover, is elevated in patients with RA with joint destruction and radiographic progression compared with controls $[8,17]$. IL-6 signaling may also influence levels of serum osteocalcin (OC), a marker of bone formation, further suggesting that modulation of this pathway may positively impact the balance of bone turnover and formation [18].

Articular inflammation also leads to the secretion of joint-destructive enzymes, (e.g., MMPs) by rheumatoid synovial fibroblasts [19]; thus, MMP substrates can be used as biomarkers of articular damage [18]. MMPcleaved fragments derived from collagens or the acutephase reactant $\mathrm{C}$-reactive protein (CRP) have been described in patients with established RA [18]. Collagen types I, II, and III are the major components of bone, cartilage, and synovium, respectively [20], and MMPcleaved fragments $(\mathrm{C} 1 \mathrm{M}, \mathrm{C} 2 \mathrm{M}$, and $\mathrm{C} 3 \mathrm{M}$, respectively) may reflect articular remodeling [20, 21]. An MMPcleaved fragment of CRP, CRPM, is also a measure of synovial inflammation [18, 22, 23].

Sarilumab is a human monoclonal antibody directed against both membrane-bound and soluble forms of IL-6R $\alpha$ [24]. Sarilumab blockade of IL-6 binding to IL$6 R \alpha$ results in inhibition of IL-6-mediated signal transduction [25]. The efficacy and safety of sarilumab in combination with methotrexate (MTX) in patients with moderate-to-severe RA and inadequate response to MTX (MTX-IR) were evaluated in the two-part (phase II (part A) and phase III (part B)) MOBILITY trial (NCT01061736) [24, 26]. In MOBILITY A and B, patients treated with sarilumab demonstrated statistically significant improvements in American College of Rheumatology $20 \%$ (ACR20) response rate at weeks 12 and 24, respectively. In MOBILITY $B$, patients treated with sarilumab also demonstrated significant improvements in least squares mean change in the Health Assessment Questionnaire-Disability Index (HAQ-DI) at week 16 and mean change in the van der Heijde modified total Sharp score (mTSS) at week 52, relative to placebo + MTX. The erosion score (ES) and joint space narrowing (JSN), components of the mTSS, were significantly reduced compared with placebo + MTX as early as week 24. Sarilumab also reduced serum levels of CRP, a marker of inflammation commonly assessed in patients with RA. Both doses of sarilumab were generally well-tolerated, and the most common treatment-emergent adverse events included 
infections, neutropenia, injection site reactions, and increased transaminases.

To better understand the mechanism through which sarilumab inhibits progression of structural damage (JSN and ES), we assessed biomarkers indicative of joint damage and bone resorption. First, biomarkers of bone and tissue destruction and synovial inflammation were measured in patients from the dose-ranging MOBILITY part A study. These analyses were then replicated and expanded upon (by including biomarkers of bone formation and resorption) in patients from the MOBILITY part B study.

\section{Methods}

\section{Study design}

The results of MOBILITY (NCT01061736), a two-part (phase II (part A) and phase III (part B)), randomized, double-blind, placebo-controlled, multicenter study that evaluated the efficacy and safety of subcutaneous sarilumab in combination with MTX in patients with active RA and MTX-IR have previously been described [24, 26]. Part A of MOBILITY was a 12-week, phase II, dose-ranging study in patients with active RA who were randomized to receive MTX in combination with placebo or one of five subcutaneous sarilumab doses [26]. Patients who participated in part A were not eligible for part B, a 52-week, phase III study evaluating the safety and efficacy of sarilumab $150 \mathrm{mg}$ and $200 \mathrm{mg}$ every 2 weeks (q2w) in combination with MTX [24].

The protocol was approved by the appropriate ethics committees/institutional review boards (see "Acknowledgments" for details), and all patients provided written informed consent before study entry. The study was conducted in compliance with institutional review board regulations, the International Conference on Harmonisation Guidelines for Good Clinical Practice, and the Declaration of Helsinki.

\section{Sera collection}

In part A, biomarkers were measured retrospectively in sera collected at baseline (i.e., before receiving the first treatment dose), and at 2 and 12 weeks posttreatment, from patients receiving placebo $+\operatorname{MTX}(n=45)$, sarilumab $150 \mathrm{mg} \mathrm{q} 2 \mathrm{w}+$ MTX $(\mathrm{n}=46)$, or sarilumab $200 \mathrm{mg}$ $q 2 w+\operatorname{MTX}(n=45)$. These doses were chosen for the present analyses as they were selected for additional efficacy and safety analyses in MOBILITY part B. Sera were collected under fasting conditions at baseline and week 12 and under nonfasting conditions at week 2. Patients were included in the analyses if at least one baseline value and at least one postbaseline value were available for one or more biomarkers under evaluation.

Biomarker analyses from part A were replicated and expanded upon in part B in sera collected under fasting conditions at baseline and 2, 24, and 52 weeks posttreatment from randomly selected patients receiving placebo + MTX $(n=128)$ or sarilumab $200 \mathrm{mg}$ q2w + MTX $(\mathrm{n}=131)$. Sarilumab $200 \mathrm{mg}$ q2w was chosen for these analyses because this dose demonstrated better efficacy compared with sarilumab $150 \mathrm{mg}$ q2w with respect to the bone and joint $x$-ray outcomes (i.e., mTSS, $\mathrm{ES}$, and JSN) in MOBILITY part B, and the additional biomarkers measured reflect pathological processes associated with these scores. To be selected for this retrospective biomarker analysis, patients were required to have baseline, week 2 , and week 24 biomarker samples and week 24 radiographic data available. Patients were included in the analyses if the baseline value and at least one postbaseline value were available for at least 1one biomarker under evaluation.

Starting at week 16 in MOBILITY part B, patients with a lack of efficacy could be "rescued" by switching to open-label sarilumab $200 \mathrm{mg}$ q2w + MTX. Patients who were rescued continued in the study according to their planned visit schedule. Samples drawn from patients in the placebo + MTX group before rescue medication were included in the biomarker analysis. Serum samples obtained from patients in the placebo + MTX group after rescue medication were excluded from the analysis.

\section{Biochemical marker assays}

Retrospective analysis of serum concentrations of C1M, C2M, C3M, and CRPM from patients from MOBILITY part A were measured at Synarc (BioClinica Laboratory, Lyon, France) using a validated proprietary enzymelinked immunosorbent assay (ELISA) by Nordic Bioscience (Herlev, Denmark). The intra-assay and inter-assay variation (coefficients of variation $(\mathrm{CVs})$ ) were $<13.8 \%$ for $\mathrm{C} 1 \mathrm{M},<19.8 \%$ for $\mathrm{C} 2 \mathrm{M},<16.4 \%$ for $\mathrm{C} 3 \mathrm{M}$, and $<14.2 \%$ for CRPM. Serum concentrations of C1M, C2M, C3M, MMP3, CTX-1, and OC from patients from MOBILITY part B were measured at Nordic Bioscience using a validated ELISA (Nordic Bioscience; all CVs $<15 \%$ ). Serum MMP-3 (Quantikine total MMP-3 (R\&D Systems, Minneapolis, $\mathrm{MN}, \mathrm{USA}) ; \mathrm{CV}<10 \%)$ and serum CTX-1 (CV $<3.4 \%)$ were measured using the $\beta$-CrossLaps (Roche, Basel, Switzerland) assay. Osteocalcin was measured using the validated N-MID-OC kit (Roche; CV $<4.6 \%$ ). Serum concentrations of sRANKL (human sRANKL ELISA (BioVendor, Brno, Czech Republic)) and OPG (human OPG ELISA (BioVendor)) were measured using validated assays at Pacific Biomarkers (Seattle, WA, USA).

\section{Statistical analysis}

Patient baseline demographics and disease parameters are presented as mean ( \pm standard deviation). Given the non-normal distribution of several biomarkers, median 
serum concentrations (quartile 1 to quartile 3 interval) were reported for baseline measures.

To evaluate differences in pharmacodynamic changes between sarilumab + MTX and placebo + MTX, a mixedeffect model with repeated measures (MMRM) was performed on rank-transformed percent change from baseline (analysis of variance (ANOVA)-type method), with the treatment, visit, and treatment-by-visit interaction included as fixed effects. Given the similar baseline biomarker values in each treatment group, baseline biomarker values were not included in the model. An MMRM was also performed on the log-transformed sRANKL/OPG ratio (to yield a normal distribution) with treatment, visit, and treatment-by-visit interaction as fixed effects, and baseline biomarker value and baseline biomarker-value-by-visit interaction as fixed covariates. An unstructured covariance structure was assumed in all models. The Bonferroni correction was used to adjust $P$ values for multiplicity. A $P$ value $<0.05$ after adjustment was considered significant.

For exploratory purposes, percent changes from baseline in biomarkers and sRANKL/OPG were also compared between responders and nonresponders (patients who achieved or did not achieve ACR50 or low disease activity (LDA), as measured by 28-joint disease activity score by CRP (DAS28-CRP) <3.2) at week 24 using similar methods and after adjustment for baseline values, separately by treatment group; nominal $P$ values are reported. Analyses were performed using SAS $^{\circ}$ v9.2 or higher (SAS Institute, Cary, NC, USA).

\section{Results}

Patient demographics, disease parameters, and baseline biomarker serum concentrations

Baseline disease characteristics in the biomarker analyses were similar to those in the overall study $[24,26]$. In part A (Table 1), the mean age of patients across all treatment groups in these biomarker analyses was 51.0 \pm 13.1 years, and patients had a mean RA duration of $7.2 \pm 7.3$ years. Patients across all treatment groups displayed similar baseline disease characteristics, including tender joint count $(27.7 \pm 16.2)$, swollen joint count $(17.7 \pm 10.8)$, and CRP concentration $(3.0 \pm 3.4 \mathrm{mg} / \mathrm{dL})$. In part B (Table 2), the mean age of patients across all treatment groups in these biomarker analyses was 50.2 \pm 11.5 years, and patients had a mean RA duration of $8.6 \pm 7.5$ years. Patients across all treatment groups displayed similar baseline disease characteristics, including tender joint count $(26.6 \pm 14.7)$, swollen joint count $(16.2 \pm 9.4)$, CRP concentration $(1.9 \pm 2.0 \mathrm{mg} / \mathrm{dL})$, and mTSS $(48.8 \pm 66.3)$. Median baseline serum concentrations of all assayed biomarkers were generally comparable across treatment groups in part A (Table 1) and part B (Table 2).

\section{Biomarkers of joint inflammation and damage}

Serum concentrations of MMP-generated biomarkers related to joint damage and tissue turnover were measured first in part A (baseline, week 2, and week 12) and subsequently in part B (baseline, week 2, and week 24). In part $\mathrm{A}$, the decrease in serum concentration of these

Table 1 Patient demographics, disease parameters, and baseline biomarker serum concentrations from MOBILITY part A biomarker analysis

\begin{tabular}{|c|c|c|c|c|}
\hline & $\begin{array}{l}\text { Placebo + MTX } \\
(\mathrm{n}=45)\end{array}$ & $\begin{array}{l}\text { Sarilumab } 150 \mathrm{mg} \text { q2w }+ \text { MTX } \\
(\mathrm{n}=46)\end{array}$ & $\begin{array}{l}\text { Sarilumab } 200 \mathrm{mg} q 2 w+\text { MTX } \\
(n=45)\end{array}$ & Total $^{a}(n=136)$ \\
\hline \multicolumn{5}{|c|}{ Baseline demographic and disease parameters } \\
\hline Age, mean $\pm S D$, years & $54.7 \pm 13.1$ & $49.8 \pm 12.7$ & $48.4 \pm 12.8$ & $51.0 \pm 13.1$ \\
\hline Sex, female, \% & 75.6 & 84.8 & 80.0 & 80.1 \\
\hline Duration of $R A$, mean $\pm S D$, years & $8.0 \pm 8.6$ & $7.1 \pm 6.7$ & $6.4 \pm 6.4$ & $7.2 \pm 7.3$ \\
\hline Anti-CCP antibody positive, $\%{ }^{\mathrm{b}}$ & 73.7 & 95.0 & 90.0 & 86.4 \\
\hline Rheumatoid factor positive, $\%$ & 66.7 & 87.0 & 88.9 & 80.9 \\
\hline Tender joint count, mean \pm SD & $27.9 \pm 17.0$ & $28.1 \pm 17.2$ & $26.9 \pm 14.6$ & $27.7 \pm 16.2$ \\
\hline Swollen joint count, mean \pm SD & $17.6 \pm 12.3$ & $18.3 \pm 10.9$ & $17.2 \pm 9.3$ & $17.7 \pm 10.8$ \\
\hline $\mathrm{CRP}$, mean $\pm \mathrm{SD}, \mathrm{mg} / \mathrm{dL}$ & $2.8 \pm 2.8$ & $2.6 \pm 2.8$ & $3.4 \pm 4.4$ & $3.0 \pm 3.4$ \\
\hline \multicolumn{5}{|c|}{ Baseline biomarker serum concentrations, median (quartile 1/quartile 3) } \\
\hline $\mathrm{C} 1 \mathrm{M}, \mathrm{ng} / \mathrm{mL}$ & $198.1(132.0 / 263.4)$ & $179.6(140.2 / 235.8)$ & $172.3(132.4 / 273.0)$ & $179.6(133.5 / 259.3)$ \\
\hline $\mathrm{C} 2 \mathrm{M}, \mathrm{ng} / \mathrm{mL}$ & $0.2(0.2 / 0.3)$ & $0.3(0.2 / 0.3)$ & $0.2(0.2 / 0.4)$ & $0.2(0.2 / 0.3)$ \\
\hline $\mathrm{C} 3 \mathrm{M}, \mathrm{ng} / \mathrm{mL}$ & $45.6(38.9 / 58.1)$ & $47.6(38.3 / 60.2)$ & $47.9(37.9 / 59.3)$ & $47.5(38.3 / 59.0)$ \\
\hline CRPM, ng/mL & $17.3(12.1 / 21.7)$ & $16.5(14.1 / 21.4)$ & $16.3(13.9 / 22.3)$ & $16.7(13.1 / 21.7)$ \\
\hline
\end{tabular}

${ }^{\mathrm{a} A l l}$ patients receiving placebo, sarilumab $150 \mathrm{mg} \mathrm{q} 2 \mathrm{w}$, or sarilumab $200 \mathrm{mg} \mathrm{q} 2 \mathrm{w}$. ${ }^{\mathrm{b}}$ Results not available for the entire biomarker population. $\mathrm{C} 1 \mathrm{M}$ collagen type I MMP-cleaved fragment, C2M collagen type II MMP-cleaved fragment, C3M collagen type III MMP-cleaved fragment, CCP cyclic citrullinated peptide, CRPM Creactive protein MMP-derived fragment, MMP matrix metalloproteinase, MTX methotrexate, $q 2 w$ every 2 weeks, $R A$ rheumatoid arthritis, $S D$ standard deviation 
Table 2 Patient demographics, disease parameters, and baseline biomarker serum concentrations from MOBILITY part B biomarker analysis

\begin{tabular}{|c|c|c|c|}
\hline & $\begin{array}{l}\text { Placebo }+ \text { MTX } \\
(n=128)\end{array}$ & $\begin{array}{l}\text { Sarilumab } 200 \mathrm{mg} \text { q2w + MTX } \\
(\mathrm{n}=131)\end{array}$ & $\begin{array}{l}\text { Total }^{\mathrm{a}} \\
(\mathrm{n}=259)\end{array}$ \\
\hline \multicolumn{4}{|c|}{ Baseline demographic and disease parameters } \\
\hline Age, mean $\pm S D$, years & $51.1 \pm 10.6$ & $49.3 \pm 12.3$ & $50.2 \pm 11.5$ \\
\hline Sex, female, \% & 77.3 & 84.7 & 81.1 \\
\hline Duration of $R A$, mean $\pm S D$, years & $9.1 \pm 8.2$ & $8.1 \pm 6.7$ & $8.6 \pm 7.5$ \\
\hline Anti-CCP antibody positive, $\%$ & 82.8 & 87.0 & 84.9 \\
\hline Rheumatoid factor positive, $\%$ & 87.5 & 87.8 & 87.6 \\
\hline Tender joint count, mean \pm SD & $27.3 \pm 14.8$ & $25.9 \pm 14.5$ & $26.6 \pm 14.7$ \\
\hline Swollen joint count, mean \pm SD & $15.8 \pm 8.0$ & $16.6 \pm 10.6$ & $16.2 \pm 9.4$ \\
\hline $\mathrm{CRP}$, mean $\pm \mathrm{SD}, \mathrm{mg} / \mathrm{dL}$ & $1.7 \pm 1.9$ & $2.1 \pm 2.1$ & $1.9 \pm 2.0$ \\
\hline mTSS, mean \pm SD & $51.8 \pm 72.1$ & $45.9 \pm 60.2$ & $48.8 \pm 66.3$ \\
\hline \multicolumn{4}{|c|}{ Baseline biomarker serum concentrations, median (quartile 1/quartile 3) } \\
\hline $\mathrm{C} 1 \mathrm{M}, \mathrm{ng} / \mathrm{mL}$ & $114.0(77.0 / 175.7)$ & $120.5(86.1 / 196.3)$ & $119.6(80.7 / 184.2)$ \\
\hline $\mathrm{C} 2 \mathrm{M}, \mathrm{ng} / \mathrm{mL}$ & $0.3(0.2 / 0.4)$ & $0.3(0.2 / 0.4)$ & $0.3(0.2 / 0.4)$ \\
\hline $\mathrm{C} 3 \mathrm{M}, \mathrm{ng} / \mathrm{mL}$ & $43.1(34.6 / 58.0)$ & $45.4(34.4 / 60.5)$ & $44.2(34.5 / 59.9)$ \\
\hline CTX-1, ng/mL & $0.4(0.3 / 0.6)$ & $0.4(0.3 / 0.5)$ & $0.4(0.3 / 0.5)$ \\
\hline $\mathrm{MMP}-3, \mathrm{ng} / \mathrm{mL}$ & $41.9(24.6 / 77.6)$ & $38.9(21.3 / 68.7)$ & $40.3(22.3 / 73.1)$ \\
\hline $\mathrm{OC}, \mathrm{ng} / \mathrm{mL}$ & $18.3(13.0 / 25.0)$ & $18.6(14.6 / 24.7)$ & $18.5(13.5 / 24.7)$ \\
\hline $\mathrm{OPG}, \mathrm{pmol} / \mathrm{L}$ & $4.9(3.9 / 6.3)$ & $5.4(3.9 / 6.7)$ & $5.2(3.9 / 6.5)$ \\
\hline sRANKL, pmol/L & $1012.5(385.0 / 3893.0)$ & $1096.0(393.0 / 2161.5)$ & $1026.0(387.0 / 2748.5)$ \\
\hline sRANKL/OPG & $245.1(64.4 / 836.5)$ & $186.3(71.8 / 401.2)$ & $212.6(70.8 / 509.7)$ \\
\hline
\end{tabular}

${ }^{a}$ All patients receiving placebo or sarilumab $200 \mathrm{mg}$ q2w. C1M collagen type I MMP-cleaved fragment, C2M collagen type II MMP-cleaved fragment, C3M collagen type III MMP-cleaved fragment, CCP cyclic citrullinated peptide, CRP C-reactive protein, CTX-1 carboxy-terminal collagen crosslinks 1, MMP matrix metalloproteinase, mTSS van der Heijde modified total Sharp score, MTX methotrexate, OC osteocalcin, OPG osteoprotegerin, $q 2 \mathrm{w}$ every 2 weeks, RA rheumatoid arthritis, SD standard deviation, sRANKL soluble receptor activator of nuclear factor-kB ligand

biomarkers from baseline was significantly greater after treatment with sarilumab 150 and $200 \mathrm{mg} \mathrm{q} 2 \mathrm{w}$ compared with placebo; suppression was numerically greater with the $200 \mathrm{mg} \mathrm{q} 2 \mathrm{w}$ dose compared with the $150 \mathrm{mg}$ $\mathrm{q} 2 \mathrm{w}$ dose. The greatest change observed was in C1M, which was significantly suppressed in patients receiving sarilumab relative to patients receiving placebo. Dosedependent decreases in C1M were observed with sarilumab treatment at week 2 (Fig. 1a); serum concentration of $\mathrm{C} 1 \mathrm{M}$ was further suppressed at week 12 in the sarilumab $150 \mathrm{mg}$ q2w group to levels observed in the $200 \mathrm{mg}$ q2w group. A $33.6 \%$ reduction from baseline was observed in the sarilumab $150 \mathrm{mg} \mathrm{q} 2 \mathrm{w}$ group at week 2 , with a $52.5 \%$ reduction from baseline observed at week $12(p<0.0001$ vs placebo for both time points). In the sarilumab $200 \mathrm{mg} \mathrm{q} 2 \mathrm{w}$ group, a $59.4 \%$ reduction from baseline at week 2 and a $61.4 \%$ reduction from baseline at week 12 was observed $(p<0.0001$ vs placebo at both time points). Treatment with placebo resulted in a $4.1 \%$ decrease from baseline over a 12-week period. In part B, circulating $\mathrm{C} 1 \mathrm{M}$ was reduced by $50.1 \%$ at week 2 and $60.3 \%$ at week 24 with sarilumab $200 \mathrm{mg} \mathrm{q} 2 \mathrm{w}$ compared with a $2.3 \%$ increase and an $8.1 \%$ reduction from baseline with placebo $(p<0.0001$ at both time points; Fig. 1b).

Modest changes in the cartilage degradation marker C2M were observed in part A. There was a $0.9 \%$ increase from baseline over the 12 weeks in the placebo group, while sarilumab reduced $\mathrm{C} 2 \mathrm{M}$ by $>10.0 \%$ by week 2 (sarilumab $150 \mathrm{mg} \mathrm{q} 2 \mathrm{w}, p<0.05$ vs placebo; sarilumab $200 \mathrm{mg} \mathrm{q} 2 \mathrm{w}, p<0.001$ vs placebo; Fig. 1c). This decrease was maintained by sarilumab $150 \mathrm{mg}$ q2w at week 12 (10.2\% decrease from baseline; $p<0.05$ vs placebo); $\mathrm{C} 2 \mathrm{M}$ was further suppressed by sarilumab $200 \mathrm{mg} \mathrm{q} 2 \mathrm{w}$ at this time point $(18.2 \%$ decrease from baseline; $p<0.001$ vs placebo). Sarilumab suppression of $\mathrm{C} 2 \mathrm{M}$ was less pronounced, and the difference relative to placebo was not observed in part B (Fig. 1d).

In part $\mathrm{A}$, sarilumab $150 \mathrm{mg} \mathrm{q} 2 \mathrm{w}$ decreased the synovial inflammation marker C3M by $18.9 \%(p<0.001$ vs placebo) and $26.6 \%$ ( $p<0.0001$ vs placebo) at weeks 2 and 12, respectively; reductions of $24.6 \%$ (week 2) and $34.9 \%$ (week 12) were observed in the sarilumab $200 \mathrm{mg} \mathrm{q} 2 \mathrm{w}(p<0.0001$ vs placebo at both time points; Fig. 1e). Similar results were observed in part B, in which C3M was reduced by $23.8 \%$ at week 2 and $31.5 \%$ 
MOBILITY PART A

a

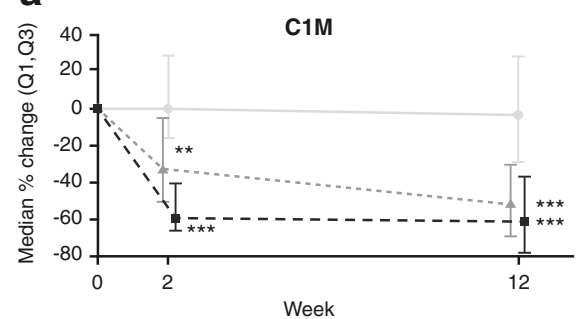

C
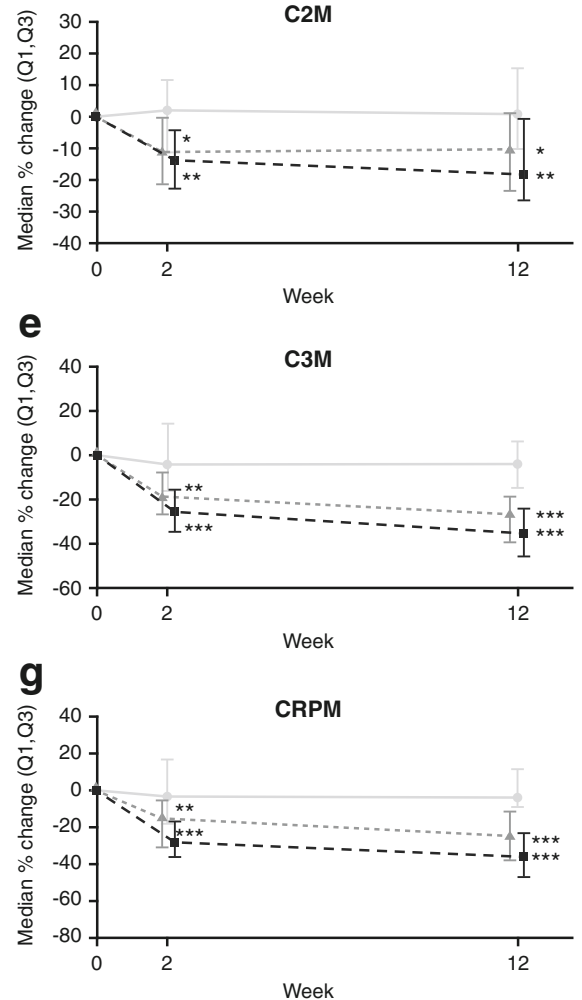

MOBILITY PART B

b

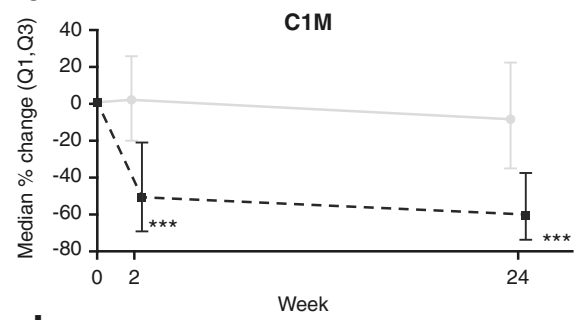

C2M
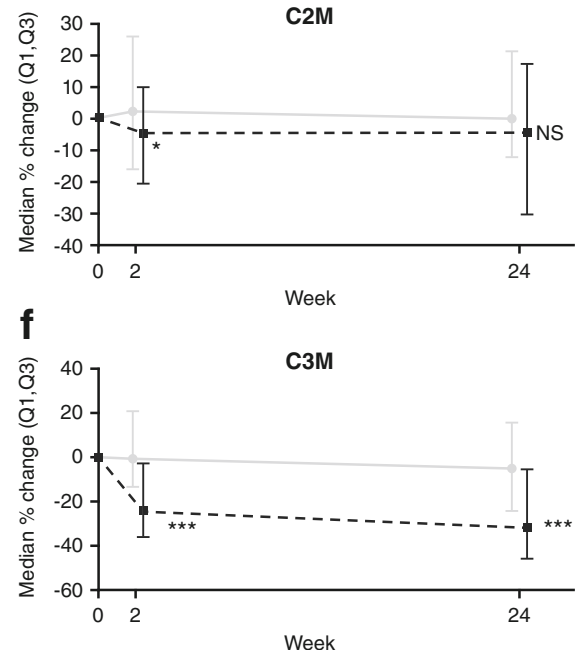

Week

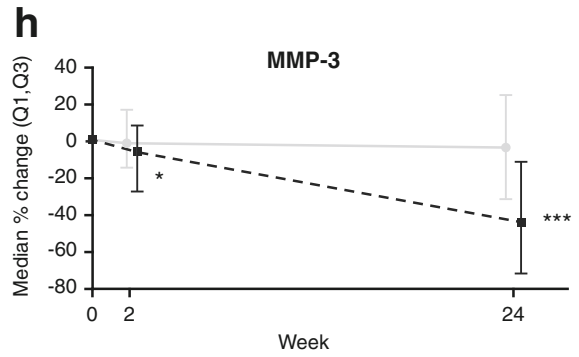

- Placebo + MTX \# Sarilumab 150 mg q2w + MTX - Sarilumab 200 mg q2w + MTX

Fig. 1 Sarilumab decreases markers of joint damage and inflammation in MOBILITY parts A and B (a and $\mathbf{b}, C 1 \mathrm{M} ; \mathbf{c}$ and $\mathbf{d}, C 2 \mathrm{M} ; \mathbf{e}$ and $\mathbf{f}, C 3 \mathrm{M} ; \mathbf{g}$, CRPM; and $\mathbf{h}, \mathrm{MMP}-3) .{ }^{*} p<0.05$ vs placebo. ${ }^{* *} p<0.01$ vs placebo. ${ }^{* * *} p<0.0001$ vs placebo. C1M collagen type I MMP-cleaved fragment, C2M collagen type II MMP-cleaved fragment, C3M collagen type III MMP-cleaved fragment, CRPM C-reactive protein MMP-derived fragment, MMP-3 matrix metalloproteinase 3, MTX methotrexate, NS not significant, (Q1,Q3) quartile 1 to quartile 3 interval, q2w every 2 weeks

at week $24(p<0.0001$ vs placebo at both time points; Fig. 1f), compared with a $5.3 \%$ reduction over 24 weeks observed with placebo.

Although placebo had minimal effects on CRPM, a marker of synovial inflammation, sarilumab reduced CRPM serum concentrations relative to baseline at weeks 2 and 12 in part A (Fig. 1g). Maximum suppression was observed at week 12 in both sarilumab groups (150 mg $\mathrm{q} 2 \mathrm{w},-25.0 \%$ from baseline; $200 \mathrm{mg} \mathrm{q} 2 \mathrm{w},-35.8 \%$ from baseline; $p<0.0001$ vs placebo for both sarilumab groups). In part $B$, significantly lower serum concentrations of MMP-3, another marker of synovial inflammation, were observed at week 2 with sarilumab 200 mg q2w compared with placebo $(-5.4 \%$ from baseline vs $-0.4 \%$ from baseline, respectively; $p<0.05$ ), and these concentrations were further decreased from baseline by week $24(-44.2 \%$ vs $-2.7 \%$, respectively; $p<0.0001$; Fig. $1 \mathrm{~h}$ ).

\section{Markers of bone resorption}

Serum concentrations of biomarkers related to bone resorption were measured in part B. Sarilumab $200 \mathrm{mg}$ $\mathrm{q} 2 \mathrm{w}$ significantly reduced sRANKL relative to placebo at week $2(p<0.05$; Fig. 2a), and sRANKL continued to decrease through week 24 in both groups, with greater suppression observed with sarilumab compared with placebo. At week 24, sarilumab 200 mg q2w significantly 


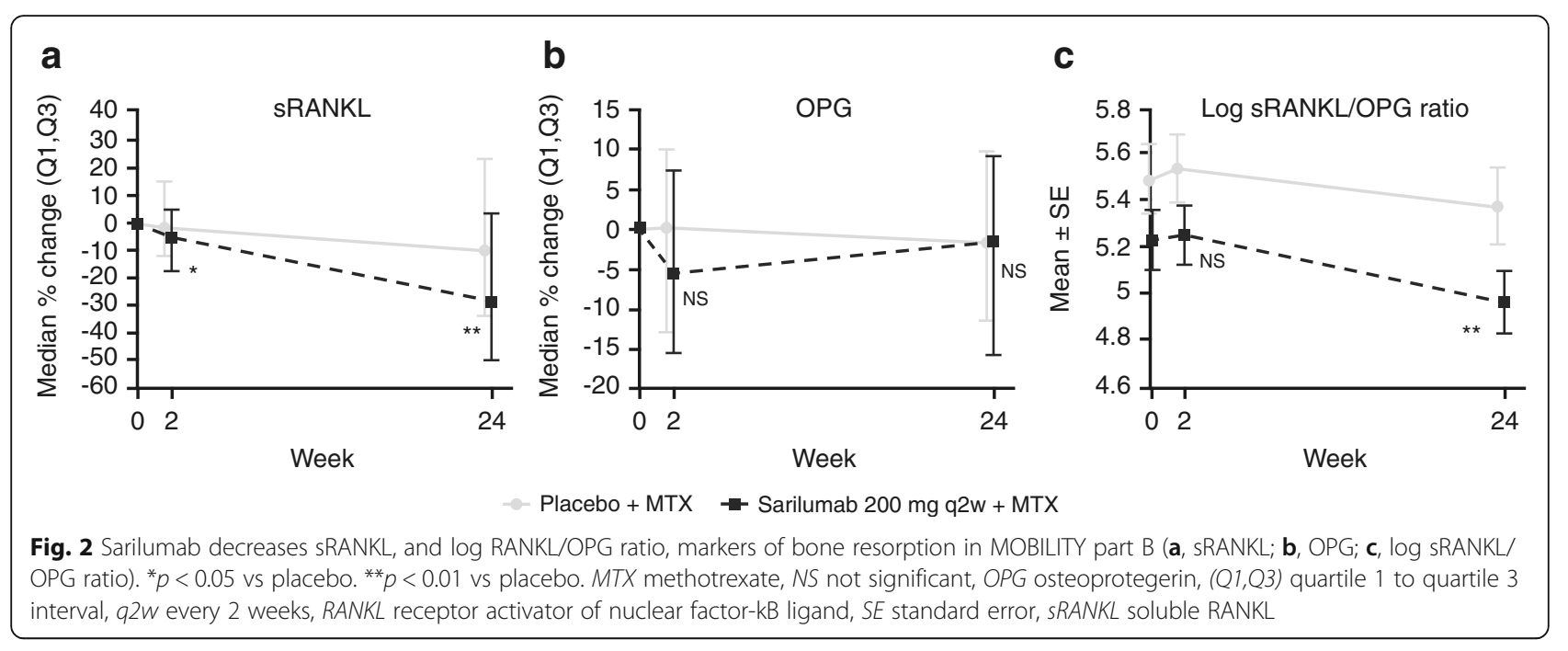

suppressed sRANKL more than placebo $(-28.6 \%$ vs $-10.2 \%$ from baseline, respectively; $p<0.01)$. No significant differences from baseline in OPG were observed in either treatment group at the time points measured (Fig. 2b). However, because of the suppressive effect of sarilumab on sRANKL, a significant decrease in the sRANKL/OPG ratio was observed in the sarilumab $200 \mathrm{mg}$ q2w group compared with placebo $(p<0.01)$ at week 24 (Fig. 2c).

Moderate reductions in CTX-1 were observed at week 24 in the sarilumab $200 \mathrm{mg} \mathrm{q} 2 \mathrm{w}$ and placebo groups (-6.7\% and $-7.8 \%$ from baseline, respectively) and week $52(-7.7 \%$ and $-7.0 \%$, respectively), but there were no significant differences between treatment groups at either time point examined (data not shown).

\section{Marker of bone formation}

Serum concentrations of $\mathrm{OC}$ were evaluated at baseline, week 24, and week 52 in samples from part B. Serum OC concentrations remained steady after treatment with placebo over the 52-week study. A numeric trend toward a larger increase in $\mathrm{OC}$ was observed with sarilumab $200 \mathrm{mg} \mathrm{q} 2 \mathrm{w}$ at week 24 (10.9\%; $p=0.107)$ and at week $52(13.2 \% ; p=0.057$, unadjusted $p=0.029)$ vs placebo ( $2.1 \%$ and $0.1 \%$, respectively), although these results were not significant after adjustment for multiplicity (Fig. 3).

\section{Biomarker changes by ACR50 response at week 24}

Percent change in serum concentrations of biomarkers were examined in ACR50 responders (placebo, $\mathrm{n}=34$ (26.6\%); sarilumab $200 \mathrm{mg} \mathrm{q} 2 \mathrm{w}, \mathrm{n}=67(51.1 \%)$ ) and nonresponders (placebo, $\mathrm{n}=94 \quad(73.4 \%)$; sarilumab $200 \mathrm{mg} \mathrm{q} 2 \mathrm{w}, \mathrm{n}=64(48.9 \%))$ at week 24 in part B (Table 3). C-reactive protein (a marker of inflammation) and markers of bone resorption and joint damage were assessed. Placebo-treated ACR50 responders demonstrated a greater reduction in CRP from baseline compared with ACR50 nonresponders, although this effect was not observed until week $8(-30.6 \%$ vs $-8.2 \%$; nominal $p<0.05)$. Only a small difference in the magnitude of CRP suppression was observed between sarilumab responders and nonresponders at this time point $(-96.6 \%$ vs $-93.3 \%$; nominal $p<0.05)$.

ACR50 responders receiving placebo demonstrated greater reductions in C1M, sRANKL, and the log sRANKL/OPG ratio at week 24 compared with placebotreated patients who did not achieve ACR50. Other biomarkers suppressed by sarilumab treatment (e.g., C3M) did not significantly differ by ACR50 response.

\section{Biomarker changes by LDA status at week 24}

Serum concentrations of biomarkers were also examined in patients who achieved LDA (placebo, $\mathrm{n}=37$ (28.9\%); sarilumab $200 \mathrm{mg} \mathrm{q} 2 \mathrm{w}, \mathrm{n}=72(55.0 \%))$ and those who did not achieve LDA (placebo, $\mathrm{n}=91$ (71.1\%); sarilumab

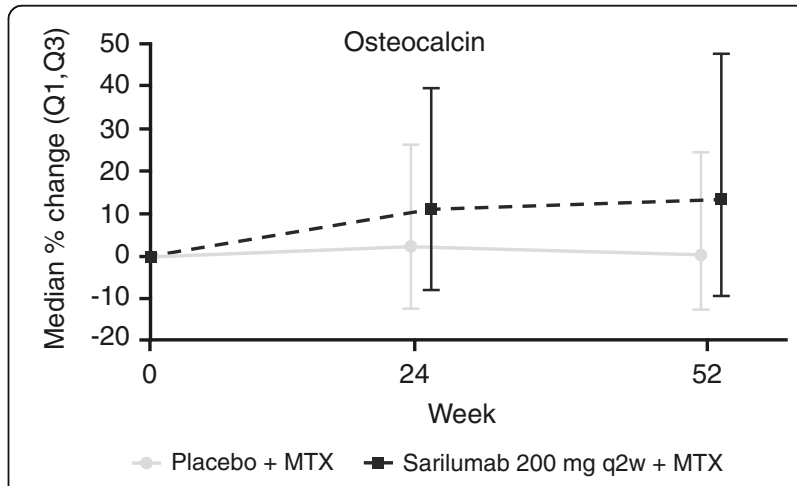

Fig. 3 Sarilumab increases OC, a marker of bone formation (nominal $p=0.057$ vs placebo, week 52). MTX methotrexate, OC osteocalcin, $(Q 1, Q 3)$ quartile 1 to quartile 3 interval, q2w every 2 weeks 
Table 3 Median percent change from baseline in biomarker concentration in ACR50 responder and nonresponder patients at week 24

\begin{tabular}{|c|c|c|c|c|}
\hline & \multicolumn{2}{|l|}{ Placebo } & \multicolumn{2}{|c|}{ Sarilumab 200 mg q2w } \\
\hline & $\begin{array}{l}\text { ACR50 } \\
\text { responder } \\
(n=34)\end{array}$ & $\begin{array}{l}\text { ACR50 } \\
\text { nonresponder } \\
(n=94)\end{array}$ & $\begin{array}{l}\text { ACR50 } \\
\text { responder } \\
(n=67)\end{array}$ & $\begin{array}{l}\text { ACR50 } \\
\text { nonresponder } \\
(n=64)\end{array}$ \\
\hline \multicolumn{5}{|l|}{ CRP } \\
\hline Week 2 & 8.5 & -3.3 & -94.2 & -87.5 \\
\hline Week 24 & $-40.3^{* *}$ & -4.2 & -96.3 & -94.1 \\
\hline \multicolumn{5}{|l|}{$\mathrm{C} 1 \mathrm{M}$} \\
\hline Week 2 & 1.6 & 2.6 & $-57.1^{*}$ & -43.8 \\
\hline Week 24 & $-26.7^{*}$ & -7.2 & $-62.8^{* *}$ & -57.0 \\
\hline \multicolumn{5}{|l|}{$\mathrm{C} 2 \mathrm{M}$} \\
\hline Week 2 & 0.0 & 3.4 & -4.3 & -4.3 \\
\hline Week 24 & 0.0 & 3.1 & 0.0 & -6.5 \\
\hline \multicolumn{5}{|l|}{ MMP-3 } \\
\hline Week 2 & -5.1 & 1.9 & $-10.8^{*}$ & -4.4 \\
\hline Week 24 & -6.3 & -1.4 & -50.9 & -30.6 \\
\hline \multicolumn{5}{|l|}{ OPG } \\
\hline Week 2 & 1.7 & 0.0 & -6.0 & -2.2 \\
\hline Week 24 & 0.0 & -1.8 & -1.1 & -2.0 \\
\hline \multicolumn{5}{|l|}{ sRANKL } \\
\hline Week 2 & -5.4 & -1.2 & -7.9 & -2.7 \\
\hline Week 24 & $-23.6^{*}$ & -0.8 & -31.4 & -24.9 \\
\hline
\end{tabular}

Percent change from baseline in biomarkers transformed in rank was compared between responder and nonresponder patients at week 24 using an analysis of variance (ANOVA)-type method, with response, visit, and response-by-visit interaction as fixed effects, rank-transformed baseline biomarker value and rank-transformed baseline biomarker-value-by-visit interaction as fixed covariates, and assuming an unstructured covariance structure. The model was run separately by treatment group (sarilumab $200 \mathrm{mg}$ q2 $\mathrm{w}$ and placebo)

ACR American College of Rheumatology

C1M collagen type I MMP-cleaved fragment, C2M collagen type II MMPcleaved fragment, CRP C-reactive protein, DAS28-CRP 28-joint disease activity score by CRP, $L D A$ low disease activity, MMP matrix metalloproteinase, $M T X$ methotrexate, OPG osteoprotegerin, $q 2 w$ every 2 weeks, sRANKL soluble receptor activator of nuclear factor-kB ligand. *Nominal $p<0.05$ vs nonresponder. ${ }^{*}$ Nominal $p<0.01$ vs nonresponder

$200 \mathrm{mg} \mathrm{q} 2 \mathrm{w}, \mathrm{n}=59(45.0 \%))$ at week 24 in part B (Table 4). Suppression of CRP according to LDA status was similar to that observed according to ACR50 response. Placebo-treated patients who achieved LDA demonstrated a greater reduction in CRP compared with patients who did not achieve LDA at week $24(-31.9 \%$ vs $-4.2 \%$; nominal $p<0.01)$ only. As with the ACR50 response analysis, the magnitude of CRP suppression observed in patients who achieved or did not achieve LDA after sarilumab treatment was only slightly different $(-96.9 \%$ vs $-90.2 \%$; nominal $p<0.01)$.

Trends for reductions in MMP-3, OPG, and sRANKL were observed in both treatment groups between patients who achieved LDA compared with patients who did not.
Table 4 Median percent change from baseline in biomarker concentration in patients who achieved or did not achieve LDA (DAS28-CRP <3.2) at week 24

\begin{tabular}{|c|c|c|c|c|}
\hline & \multicolumn{2}{|l|}{ Placebo } & \multicolumn{2}{|c|}{ Sarilumab 200 mg q2w } \\
\hline & $\begin{array}{l}\text { LDA } \\
\text { achieved } \\
(n=37)\end{array}$ & $\begin{array}{l}\text { LDA not } \\
\text { achieved } \\
(n=91)\end{array}$ & $\begin{array}{l}\text { LDA } \\
\text { achieved } \\
(n=72)\end{array}$ & $\begin{array}{l}\text { LDA not } \\
\text { achieved } \\
(n=59)\end{array}$ \\
\hline \multicolumn{5}{|l|}{ CRP } \\
\hline Week 2 & 1.2 & -2.9 & $-95.0^{* *}$ & -83.5 \\
\hline Week 24 & $-31.9^{* *}$ & -4.2 & $-96.9^{* *}$ & -90.2 \\
\hline \multicolumn{5}{|l|}{$\mathrm{C} 1 \mathrm{M}$} \\
\hline Week 2 & 4.5 & 2.2 & $-55.8^{*}$ & -45.1 \\
\hline Week 24 & $-16.8^{*}$ & -4.3 & $-65.7^{* *}$ & -54.1 \\
\hline \multicolumn{5}{|l|}{$\mathrm{C} 2 \mathrm{M}$} \\
\hline Week 2 & 0 & 3.4 & $0^{*}$ & -14.3 \\
\hline Week 24 & 0 & 3.5 & 0 & -6.7 \\
\hline \multicolumn{5}{|l|}{ MMP-3 } \\
\hline Week 2 & -2.0 & 2.3 & -7.2 & -3.3 \\
\hline Week 24 & -9.7 & 0.4 & -47.2 & -34.2 \\
\hline \multicolumn{5}{|l|}{ OPG } \\
\hline Week 2 & -5.1 & 1.6 & $-6.5^{*}$ & -0.9 \\
\hline Week 24 & -1.9 & -1.8 & -4.6 & 0.9 \\
\hline \multicolumn{5}{|l|}{ sRANKL } \\
\hline Week 2 & -4.7 & -0.8 & -7.9 & -2.3 \\
\hline Week 24 & $-16.2^{*}$ & -0.8 & -39.7 & -25.8 \\
\hline
\end{tabular}

Percent change from baseline in biomarkers transformed in rank was compared between responder and nonresponder patients at week 24 using an analysis of variance (ANOVA)-type method, with response, visit, and response-by-visit interaction as fixed effects, rank-transformed baseline biomarker value and rank-transformed baseline biomarker-value-by-visit interaction as fixed covariates, and assuming an unstructured covariance structure. The model was run separately by treatment group (sarilumab $200 \mathrm{mg} \mathrm{q} 2 \mathrm{w}$ and placebo).

C1M collagen type I MMP-cleaved fragment, C2M collagen type II MMPcleaved fragment, CRP C-reactive protein, DAS28-CRP 28-joint disease activity score by CRP, LDA low disease activity, MMP matrix metalloproteinase, MTX methotrexate, OPG osteoprotegerin, $q 2 w$ every 2 weeks, sRANKL soluble receptor activator of nuclear factor-kB ligand. *Nominal $p<0.05$ vs nonresponder. **Nominal $p<0.01$ vs nonresponder

Most of the differences were not significant with the exception of $\mathrm{C} 1 \mathrm{M}$, which was reduced in placebo-treated and sarilumab-treated patients. C3M reduction was not different between patients who did or did not achieve LDA, despite suppression by sarilumab treatment (data not shown).

\section{Discussion}

The effects of treatment with sarilumab plus MTX on biomarkers of joint and tissue destruction and bone resorption were examined in MTX-IR patients with RA from the MOBILITY trial. Given the reduction in the progression of structural damage observed in patients receiving sarilumab 150 or $200 \mathrm{mg} \mathrm{q} 2 \mathrm{w}$ (particularly in those receiving $200 \mathrm{mg} \mathrm{q} 2 \mathrm{w}$ ) [24], blockade of IL-6R $\alpha$ with this antibody was predicted to significantly impact 
serum concentrations of biomarkers of joint and tissue destruction and bone resorption. Consistent with this prediction, sarilumab significantly reduced concentrations of markers of joint inflammation (e.g., C3M and MMP-3) and collagen degradation (C2M) compared with placebo. A rapid reduction in several MMP-generated biomarkers was observed as early as 2 weeks after initiation of sarilumab, was sustained for at least 24 weeks, and was dose dependent. Significant correlations between baseline concentrations of $\mathrm{C} 1 \mathrm{M}$, a marker of soft tissue destruction, have previously been observed with CRP concentrations and structural damage in MTX-IR patients with RA, indicating the potential prognostic utility of this marker [27].

Reductions in MMP-3 (stromelysin-1), a marker of synovial inflammation, were also observed at week 2 , with continuing reductions observed at week 24 , in patients treated with sarilumab compared with those treated with placebo. MMP-3 is highly elevated in the joint tissue and synovial fluid of patients with RA [19, 28], and higher baseline concentrations of this enzyme are associated with disease activity and radiographic progression, particularly in individuals with early RA (i.e., duration of symptoms $<12$ months) [19].

Serum concentrations of a separate marker of synovial inflammation, CRPM, were also reduced at weeks 2 and 12 in patients treated with sarilumab compared with placebo. Maximum suppression was observed at week 12 in the sarilumab 150 and $200 \mathrm{mg}$ q2w groups, although the mechanism underlying this reduction remains uncertain. Previous reports from the MOBILITY study have shown that sarilumab significantly decreases CRP [24, 26]; as such, the reduction in CRPM observed in the present study could be due to a decrease in proteolysis and/or a decrease in substrate available for MMP-mediated cleavage.

Sarilumab was also associated with a trend toward an increase in OC, a marker of bone formation, in the MTXIR patient population. Together, the data in the present report are consistent with other studies, in which blockade of IL-6R with tocilizumab was associated with reduced circulating serum concentrations of MMP-3 and MMP-3cleaved fragments, including $\mathrm{C} 1 \mathrm{M}, \mathrm{C} 2 \mathrm{M}, \mathrm{C} 3 \mathrm{M}$, and CRPM, and augmentation of OC [18, 27, 29].

Importantly, this placebo-controlled study reported that an inhibition of IL-6 signaling leads to significant sRANKL reduction in patients with MTX-IR RA, which may indicate a potential mechanism through which inhibition of IL-6 signaling prevents further progression of bone resorption and loss in this patient population. Previous work has shown that RANKL concentration is negatively correlated with bone mineral density (BMD) in patients with RA $[30,31]$ and can be blocked by antiRANKL monoclonal antibodies that increase BMD, such as denosumab [32]. Furthermore, in patients with refractory
RA who received anti-tumor necrosis factor (TNF) therapy, sRANKL serum concentrations of RANKL have been suggested as potential predictive markers of remission [33].

Sarilumab did not significantly affect serum concentrations of OPG, a decoy receptor for RANKL that negatively regulates osteoclast maturation, compared with placebo [34]. This is in contrast with previous observations, in which patients with RA who had received treatment with tocilizumab demonstrated enhanced bone marrow OPG expression relative to patients with RA who had not received biologic therapy [35]. However, in the present analysis of patients with moderate-to-severe RA, blockade of IL-6R $\alpha$ with sarilumab significantly decreased the sRANKL/OPG ratio, which is often used to measure the magnitude of bone resorption [16] and has been shown to predict 5-year and 11-year joint damage in patients with early untreated RA $[16,36]$. The current data also support and expand upon previous work, in which blockade of IL-6R with tocilizumab significantly impacted bone resorption, particularly in patients achieving remission or low disease activity [37].

Although sarilumab significantly suppressed one marker of bone resorption, sRANKL, sarilumab did not significantly modulate CTX-1 relative to placebo at either time point examined. In a previous investigation, treatment with tocilizumab had variable effects on serum concentrations of CTX-1 [18]. In the present study, serum concentrations of CTX-1 were measured at baseline, week 24, and week 52; as there may be a temporal relationship between IL-6R $\alpha$ blockade and CTX-1 suppression, further analysis at earlier time points may be warranted. CTX-1 is created through cathepsin $\mathrm{K}$ cleavage of collagen type I $[20,38]$. The lack of CTX-1 modulation observed in the present study suggests that sarilumab may not impact cathepsin K cleavage of collagen type I and may only impact MMP cleavage as reflected in reduction of C1M.

Although posttreatment differences were observed in several biomarkers according to clinical response, most of the significant differences relating to ACR50 response were noted in the placebo group. Modest differences were observed earlier in responders to sarilumab treatment. Additional analysis is needed to determine if the baseline biomarkers or changes in biomarkers can predict clinical response to sarilumab.

The present investigation retrospectively evaluated serum biomarker concentrations collected as part of two independent, randomized, placebo-controlled trials, in which patients with RA received placebo or sarilumab with concomitant MTX. This design not only permitted direct comparison between treatment groups but also allowed for analysis of duration of biomarker responses. This study design also provided a unique opportunity to replicate biomarker assessments in two independent cohorts of similar populations of MTX-IR patients with 
moderate-to-severe, active RA. The reductions observed in markers of tissue destruction $(\mathrm{C} 1 \mathrm{M})$, cartilage destruction $(\mathrm{C} 2 \mathrm{M})$, and synovial inflammation $(\mathrm{C} 3 \mathrm{M})$ in part A were also observed in part B. Evaluation of joint damage progression in MOBILITY part $\mathrm{B}$ provided the basis to also assess biomarkers of bone resorption (CTX1, OPG, sRANKL, and sRANKL/OPG ratio) and formation $(\mathrm{OC})$. Future analysis will involve correlation of these markers with the radiographic efficacy endpoints (i.e., mTSS, ES, JSN) measured in the main study.

Despite these advantages, there are several limitations. First, only circulating markers of joint damage and resorption were examined. Future studies are needed to examine the effect of sarilumab levels on these markers in the synovial fluid or in synovial tissue. Second, biomarkers were evaluated at a limited number of time points after treatment. Dose-dependent effects observed in MOBILITY part A were not evaluated in MOBILITY part B, which only evaluated the effects of the sarilumab $200 \mathrm{mg}$ q $2 \mathrm{w}$ dose. Finally, the current analyses do not take into consideration concomitant medications (e.g., corticosteroids [24]) that may possibly influence expression of biomarkers examined in the present study (e.g., RANKL [39]). Therefore, further analyses are needed to clarify the effect on these biomarkers of sarilumab vs concomitant medication vs their combination.

These data are consistent with previous studies and provide additional evidence of reduction in markers of bone resorption in response to sarilumab treatment. Overall, the study findings support a model wherein IL-6 signaling inhibition reduces osteoclast-driven structural damage and reduces joint inflammation markers in patients with established RA and MTX-IR.

\section{Conclusions}

The present investigation demonstrated the pharmacodynamic effects of sarilumab plus MTX on serum concentrations of biomarkers associated with joint and tissue destruction and damage compared with placebo plus MTX. In the future, quantitative measurements of biomarkers of bone and cartilage damage may be useful as prognostic markers to identify patients most in need of treatment. Additionally, such measurements may also serve as predictive markers of positive responses to IL-6 inhibition, allowing interpretation of potential treatment efficacy earlier than radiological-based measurements [40]. To explore the potential role of biomarkers of joint and tissue destruction and inflammation in therapeutic targeting, additional studies are necessary to determine whether serum concentrations of these biomarkers of joint damage, bone resorption, and synovial inflammation can serve as early identifiers of both severe disease and patients likely to respond to sarilumab.

\section{Abbreviations}

ACR: American College of Rheumatology; ANOVA: Analysis of variance;

C1M: Collagen type I matrix metalloproteinase-cleaved fragment;

C2M: Collagen type II matrix metalloproteinase-cleaved fragment;

C3M: Collagen type III matrix metalloproteinase-cleaved fragment; CRP: Creactive protein; CRPM: C-reactive protein matrix metalloproteinase-derived fragment; CTX-1: Collagen type I matrix metalloproteinase-cleaved fragment C-telopeptide 1; DAS28-CRP: 28-joint disease activity score by C-reactive protein; ELISA: Enzyme-linked immunosorbent assay;

ES: erosion score; FLS: Fibroblast-like synoviocytes; IL-6: Interleukin 6; IL6R: Interleukin 6 receptor; JSN: Joint space narrowing; LDA: Low disease activity; MMP: Matrix metalloproteinase; mTSS: van der Heijde modified total Sharp score; MTX: Methotrexate; MTX-IR: Inadequate response to methotrexate; OC: Osteocalcin; OPG: Osteoprotegerin; q2w: Every 2 weeks; RA: Rheumatoid arthritis; RANKL: Receptor activator of nuclear factor-kB ligand; sRANKL: Soluble receptor activator of nuclear factor-kB ligand; TNF: Tumor necrosis factor

\section{Acknowledgements}

This study was sponsored by Sanofi and Regeneron Pharmaceuticals, Inc. Editorial assistance was provided under the direction of the authors by Gretchen Chidester, PhD, MedThink SciCom, and funded by Sanofi and Regeneron Pharmaceuticals, Inc. The authors thank Dr Emmanuelle Cousin, Ling Cai, and Xin Zhang for their assistance with data analysis and interpretation and Drs Hubert van Hoogstraten and Paul Cavanaugh of Sanofi for their critical review of the manuscript. The specific ethical bodies that approved study EFC11072-part B are as follows: CIE Para Ensayos en Farmacologia Clin Prof Dr Luis M. Zieher Jose E. Uriburu, Buenos Aires; CIEIS Italiano De Cordoba Roma, Córdoba; Comité de Etica Instituto Strusberg, Córdoba; CIEM-NOA Las Piedras, Tucumán; CIEFA Uruguay, Buenos Aires; Instituto De Investigaciones Clínicas Zarate F. Andrade, Buenos Aires Province; Dim Clínica Privada Belgrano, Buenos Aires Province; CECIC - Comité de Ética de CER Investigaciones Clínicas Vicente Lopez, Buenos Aires Province; Instituto De Investigaciones Clínicas Avda, Buenos Aires Province; RedcliffeCaboolture Health Service District Ethics Committee, Queensland; Sydney Local Health Network (SLHN) Ethics Review Committee, New South Wales; Austin Health Human Research Ethics Committee Austin Hospital, Victoria; Human Research Ethics Committee (TQEH/LMH/MH) The Queen Elizabeth Hospital, South Australia; Royal Brisbane Hospital EC Royal Brisbane Hospital \& Women's Hospital Health, Queensland; ACT Health Human Research Ethics Committee Canberra Hospital, Australian Capital Territory; Bellberry Human Research Ethics Committees, South Australia; Ethikkommission der Medizinischen Universität Graz, Graz; EK der Stadt Wien gemäß KAG, AMG und MPG Town, Wien; CHU de Liège Domaine Universitaire du Sart-Tilman, Liège; EC of Hospital das Clínicas da Univ. Federal do Parana Rua General Carneiro, Parana; Comissão Nacional de Ética em Pesquisa - CONEP Edifício Ex-INAN - Unidade II, Brasilia; EC of Instituto de Assistancia Medica Servidor Publico Estadual, Sao Paulo; Ethics Committee of Hospital Heliopolis Rua Conego Xavier, Sao Paulo; EC of Pontifícia Universidade Católica do Rio Grande do Sul, Rio Grande do Sul; EC of Hospital Universitario Pedro Ernesto, Rio de Janeiro; Ethics Committee of Hospital Geral de Goiania, Goias; EC of Pontifícia Univ. Católica de Campinas, Sao Paulo; EC of Hospital Universitario da Univ Federal de Juiz de Fora, Minas Gerais; Ethics Committee Prof. Dr. Celso Figueiroa SCMBA Praca Conselheiro Almeida Couto, Bahia; EC of Faculdade de Ciencias Medicas UNICAMP, Sao Paulo; EC of Centro Integrado de Atencao a Saude - UNIMED Vitoria, Espirito Santo; Local Ethics Committee of the City Clinical Hospital Number 9, Minsk; LEC of the Clinical Hospital 6, Minsk; Institutional Review Board Services, Aurora; University Health Network Research Ethics Board, Toronto; Comité de Ética de la Investigacion S.S.M Centra, Santiago; Comité de Ética Científico S.S. M Oriente, Santiago; Comité Etico de Investigacion Servicio de Salud Valdivia, Valdivia; Comité de Ética de la Investigacion S.S. Viña Quillota, Vina Del Mar; Comité de Ética de la Investigacion S.S.M Sur Oriente, Santiago; Comité de Ética de la Investigacion S.S.M. Sur; Santiago; Comité de Ética Cientifica del Servicio de Salud del Maule, Talca; National Taiwan University Hospital 7, Taipei; Chang Gung Memorial Hospital (Institutional Review Board) Chang Gung Memorial Hospital No5, Taoyuan; CIE en Investigacion del H. Militar Central, Bogotá; Comité de Investigaciones y Etica en Investigaciones, Medellin; Comité de Etica en Investigacion de la Universidad del Norte, Barranquilla; Comité de Ética de la Investigacion Riesgo de Fractura, Bogotá; Comité de Ética en Investigaciones del Oriente, Bucaramanga; Fundacion Instituto de Reumatologia Fernando Chalem, Bogotá; Comité de Etica en Investigacion de Servimed EU, Bucaramanga; 
Tallinn Medical Research Ethics Committee, Tallinn; HUS Medisiininen Eettinen Toimikunta Biomedicum Helsinki 2 C, Helsinki; Ethik-Kommission der Ärztekammer Westfalen-Lippe und der Medizinischen Fakultät der Westfälischen Wilhelms-Universität Münster, Münster; Fachbereichs Medizin der Johann Wolfgang Goethe-Universität Frankfurt, Frankfurt am Main; EthikKommission der Medizinischen Fakultät Friedrich-Alexander-Universität Erlangen-Nürnberg, Erlangen; Ethik-Kommission der Ärztekammer Hamburg, Hamburg; Ärztekammer Niedersachsen Ethikkommission zur Beurteilung Medizinischer Forschung am Menschen, Hannover; Ethik-Kommission des Landes Berlin Landesamt für Gesundheit und Soziales Berlin, Berlin; EthikKommission I der Medizinischen Fakultät Heidelberg, Heidelberg; EthikKommission des Landes Sachsen-Anhalt, Dessau-Rosslau; National Ethics Committee 284, Messoghion, Athens; Medical Research Council, Ethics Committee for Clin. Pharm., Budapest; Center for Rheumatic Diseases (CRD) Ethics Committee CRD, 11, Hermes Elegance, Pune; Medanta Independent Ethics Committee Medanta - The Medicity, Gurgoan; CMMH National Ethics Committee National Ethics Committee, Chennai; IEC of Sri Deepti Rheumatology Center Sri Deepti Rheumatology Center, Hyderabad; Institution Ethics Committee \#149, Bangalore; Penta Med Ethics Committee Medipoint Hospitals PVT LTD, Pune; KIMS Institutional Ethics Committee, Secunderabad; Sanjay Gandhi Postgraduate Institute of Medical Sciences, Lucknow; Gachon University Gil Hospital IRB, Incheon; CMC Central IRB, Seoul; Institutional Review Board of the Catholic University of Korea, Seoul; Institutional Review Board of Kyungpook Nat'I Univ. Hospital, Daegu; IRB of Eulji University Hospital, Daejeon; Pusan National University Hospital, Busan; Institutional Review Board of National University Hospital, Seoul; Ajou University Hospital, Gyeonggi-do; Institutional Review Board of Inha University Hospital, Incheon; Institutional Review Board of Chonnam National University Hospital, Gwangju; Institution Review Board of Chonbuk National University Hosp, Jeollabuk-do; The Hospital for Rheumatic Disease Hanyang University, Seoul; Institutional Review Board of Hallym Sacred Heart Hospital, Gyeonggi-do; Medical Research \& Ethics Committee C/o Institute for Health Management, Kuala Lumpur; Comité Bioetico para la Investigacion Clínica S.C., México, D.F.; CEl Centro de Estudios de Investigación Básica y Clínica SC, Jalisco; CEl Centro de Especialidades Médicas del Sureste, S.A.deC.V., Yucatan; Comité de Ética Faculdad de Medicina de la UANL y HU, Nuevo Leon; Comité de Ética de la F. de Med. de la UAEM Paseo Tollocan S/N Col., Estado de México; Comision de Investigacion Etica y Bioseguridad Cen. Esp. en Diab., Ob. y Prev. de Enf. Cardiovasc., SC, México, D.F.; CEl Unidad de Atención Médica e Invest. en Salud (UNAMIS), Yucatan; Multi-region Ethics Committee NZ Multi-region EC, Wellington; Regional Komité for Medisinsk og Helsefaglig Forskningsetikk, Oslo; Institutional Review Board-University of Sto. Tomas Hospital University of Sto. Tomas Hospital, Manila; UP PGH-RIDO Philippine General Hospital, Manila; Cebu Doctors University Institutional Ethics Review Committee, Cebu; Komisja Bioetyczna przy Kujawsko-Pomorskiej Okr. Izbie Lekar, Kujawsko-pomorskie; CEIC - Comissão de Ética para a Investigação Clínica, Lisboa; National Ethics Committee for Clinical Trial of the Medicine, Bucharest; LEC of the Scientific Research Institute of Rheumatology, Moscow; National Ethics Committee of the Russian Federation, Moscow; Russian State Medical University, Moscow; LEC of Aviation Clinical Hospital \#7, Moscow; LEC of the Kemerovo State Medical Academy, Kemerovo; LEC of the Hospital \#25, Saint-Petersburg; LEC of t Dzhanelidze Research Institute, Saint-Petersburg; LEC of Immunology Research Institute, Novosibirsk; LEC of Samara Region Hospital named after M.I. Kalinin, Samara; LEC of the Saratov State Medical University, Saratov; LEC of the Central Clinical Hospital with Outpatient Department, Moscow; LEC of Republican Clinical Hospital named by G.G. Kuvatov, Ufa; LEC of the City Clinical Hospital\#26, Saint-Petersburg; Ryazan Regional Clinical Cardiological Dispensary, Ryazan; LEC of the Kemerovo Regional Clinical Hospital, Kemerovo; LEC of 'Applied Medicine,' Moscow; Wits Human Research EC, Gauteng; Pharma Ethics (Pty) Ltd, Pretoria; University of Kwazulu-Natal, Biomedical Research Ethics Committee, Durban; The Research Ethics Committee, University of Pretoria Faculty of Health Sciences, University of Pretoria, Pretoria Gauteng; CEIC Hospital Virgen Macarena, Sevilla; The Ethical Clearance Committee on Human Rights Related to Research Involving Human Subjects Ramathibodi Hospital, Bangkok; The Ethical Committee on Research Involving Human Subject Siriraj Hospital, Bangkok; LEC of Central City Clinical Hospital \#1, Donetsk; LEC of Railway Clinical Hospital, Dnipropetrovsk; LEC of "M.D. Strazhesko Institute of Cardiology of AMS of Ukraine," Kyiv; LEC of City Clinical Hospital \#4, Lviv; LEC of "University Clinic," Simferopol; LEC of Oleksandrivska Clinical Hospital, Kyiv; LEC of Municipal Institution "Zaporizhzhya Regional Clinical Hospital," Zaporizhzhya; LEC of Kharkov Regional Clinical
Hospital, Khark; Compass IRB, Mesa, AZ; Emory University IRB, Atlanta, GA; Schulman Associates Institutional Review Board, Cincinnati, OH; IRB University of Florida Health Center Institutional Review Board, Gainesville, FL; New York University School of Medicine IRB, New York, NY; Ethics Committee of Centro de Ciencias da Saude UFPE, Pernambuco; Ethik-Kommission der Landesärztekammer in Hessen, Frankfurt; Ethics Committee for Research on Human Subject Seth GS Medical College and KEM Hospital, Mumbai; Ethics Committee on Clinical Trials Indraprastha Apollo Hospitals, Delhi; Institutional Ethics Committee CSSMU Office of the Research Cell of C.S.M. Medical University, Lucknow; Ethics Committee of Care Institute of Medical Sciences CIMS Hospitals, Ahmedabad; Daegu Catholic University Medical Center, Daegu-si; Ministry of Health Ethics Committee, Ankara; Research Ethics Committee Faculty of Medicine Cairo University, Cairo; WIRB, Olympia, WA; University of California, San Diego IRB, La Jolla, CA; University of Texas Southwestern Medical Center IRB, Dallas, TX.

\section{Funding}

This study was sponsored by Sanofi and Regeneron Pharmaceuticals, Inc. Funding for editorial assistance in the preparation of the manuscript was also provided by Sanofi and Regeneron Pharmaceuticals, Inc.

\section{Authors' contributions}

$A B$ contributed to conception and design of study; acquisition, analysis, and interpretation of data; and drafting and critically revising the manuscript for important intellectual content. JM contributed to acquisition, analysis, and interpretation of data; and drafting and critically revising the manuscript for important intellectual content. SF contributed to design of study and critically revising the manuscript for important intellectual content. JVA contributed to conception of study; interpretation of data; and drafting and critically revising the manuscript for important intellectual content. NMHG contributed to conception of study and drafting and critically revising the manuscript for important intellectual content. JDH contributed to conception and design of study and drafting and critically revising the manuscript for important intellectual content. All authors have approved this manuscript for publication.

\section{Competing interests}

$A B, J V A, N M H G$, and JDH are employees of Regeneron Pharmaceuticals, Inc, and may hold stock and/or stock options in the company. JM and SF are employees of Sanofi and may hold stock and/or stock options in the company. The authors declare that they have no competing interests.

\section{Ethics approval and consent to participate}

The protocol was approved by the appropriate ethics committees/institutional review boards (see Acknowledgments for details), and all patients provided written informed consent before study entry. The study was conducted in compliance with institutional review board regulations, the International Conference on Harmonisation Guidelines for Good Clinical Practice, and the Declaration of Helsinki.

\section{Disclosures}

This research was sponsored by Regeneron Pharmaceuticals, Inc, and Sanofi.

\section{Author details}

${ }^{1}$ Regeneron Pharmaceuticals, Inc., 777 Old Saw Mill River Road, Tarrytown, NY 10591, USA. 'Sanofi R\&D, 1 Avenue Pierre Brossolette, 91380 Chilly-Mazarin, France. ${ }^{3}$ Sanofi US, 55 Corporate Drive, Bridgewater, NJ 08807, USA.

Received: 22 March 2016 Accepted: 20 September 2016 Published online: 06 October 2016

\section{References}

1. Schett G, Hayer S, Zwerina J, Redlich K, Smolen JS. Mechanisms of disease: the link between RANKL and arthritic bone disease. Nat Clin Pract Rheumatol. 2005;1:47-54.

2. Scott DL, Wolfe F, Huizinga TW. Rheumatoid arthritis. Lancet. 2010;376:1094-108.

3. Scott DL, Pugner K, Kaarela K, Doyle DV, Woolf A, Holmes J, et al. The links between joint damage and disability in rheumatoid arthritis. Rheumatology (Oxford). 2000;39:122-32.

4. Srirangan S, Choy EH. The role of interleukin 6 in the pathophysiology of rheumatoid arthritis. Ther Adv Musculoskelet Dis. 2010;2:247-56. 
5. Houssiau FA, Devogelaer JP, Van Damme J, de Deuxchaisnes CN, Van Snick J. Interleukin-6 in synovial fluid and serum of patients with rheumatoid arthritis and other inflammatory arthritides. Arthritis Rheum. 1988;31:784-8.

6. Ishimi Y, Miyaura C, Jin CH, Akatsu T, Abe E, Nakamura Y, et al. IL-6 is produced by osteoblasts and induces bone resorption. J Immunol. 1990; 145:3297-303

7. Kotake S, Sato K, Kim KJ, Takahashi N, Udagawa N, Nakamura I, et al. Interleukin-6 and soluble interleukin-6 receptors in the synovial fluids from rheumatoid arthritis patients are responsible for osteoclast-like cell formation. J Bone Miner Res. 1996;11:88-95.

8. Garnero P, Jouvenne P, Buchs N, Delmas PD, Miossec P. Uncoupling of bone metabolism in rheumatoid arthritis patients with or without joint destruction: assessment with serum type I collagen breakdown products. Bone. 1999;24:381-5.

9. Hashizume M, Hayakawa N, Mihara M. IL-6 trans-signalling directly induces RANKL on fibroblast-like synovial cells and is involved in RANKL induction by TNF- $a$ and IL-17. Rheumatology (Oxford). 2008;47:1635-40.

10. Tamura T, Udagawa N, Takahashi N, Miyaura C, Tanaka S, Yamada Y, et al. Soluble interleukin-6 receptor triggers osteoclast formation by interleukin 6 . Proc Natl Acad Sci U S A. 1993;90:11924-8.

11. Udagawa N, Takahashi N, Katagiri T, Tamura T, Wada S, Findlay DM, et al. Interleukin (IL)-6 induction of osteoclast differentiation depends on IL-6 receptors expressed on osteoblastic cells but not on osteoclast progenitors. J Exp Med. 1995;182:1461-8.

12. Kudo O, Sabokbar A, Pocock A, Itonaga I, Fujikawa Y, Athanasou NA. Interleukin-6 and interleukin-11 support human osteoclast formation by a RANKL-independent mechanism. Bone. 2003;32:1-7.

13. Hofbauer LC, Heufelder AE. The role of osteoprotegerin and receptor activator of nuclear factor $\mathrm{kB}$ ligand in the pathogenesis and treatment of rheumatoid arthritis. Arthritis Rheum. 2001;44:253-9.

14. Axmann R, Böhm C, Krönke G, Zwerina J, Smolen J, Schett G. Inhibition of interleukin-6 receptor directly blocks osteoclast formation in vitro and in vivo. Arthritis Rheum. 2009;60:2747-56.

15. Kato A, Matsuo S, Takai H, Uchiyama Y, Mihara M, Suzuki M. Early effects of tocilizumab on bone and bone marrow lesions in a collagen-induced arthritis monkey model. Exp Mol Pathol. 2008;84:262-70.

16. Geusens PP, Landewé RB, Garnero P, Chen D, Dunstan CR, Lems WF, et al. The ratio of circulating osteoprotegerin to RANKL in early rheumatoid arthritis predicts later joint destruction. Arthritis Rheum. 2006;54:1772-7.

17. Syversen SW, Goll GL, van der Heijde D, Landewé R, Gaarder PI, Odegård S, et al. Cartilage and bone biomarkers in rheumatoid arthritis: prediction of 10-year radiographic progression. J Rheumatol. 2009;36:266-72.

18. Bay-Jensen AC, Platt A, Byrjalsen I, Vergnoud P, Christiansen C, Karsdal MA. Effect of tocilizumab combined with methotrexate on circulating biomarkers of synovium, cartilage, and bone in the LITHE study. Semin Arthritis Rheum. 2014:43:470-8.

19. Green MJ, Gough AK, Devlin J, Smith J, Astin P, Taylor D, et al. Serum MMP3 and MMP-1 and progression of joint damage in early rheumatoid arthritis. Rheumatology (Oxford). 2003;42:83-8.

20. Garnero P, Geusens P, Landewé R. Biochemical markers of joint tissue turnover in early rheumatoid arthritis. Clin Exp Rheumatol. 2003;21 Suppl 31:S54-8.

21. Bay-Jensen AC, Leeming DJ, Kleyer A, Veidal SS, Schett G, Karsdal MA. Ankylosing spondylitis is characterized by an increased turnover of several different metalloproteinase-derived collagen species: a cross-sectional study. Rheumatol Int. 2012;32:3565-72.

22. Siebuhr AS, Petersen KK, Arendt-Nielsen L, Egsgaard LL, Eskehave T, Christiansen $\mathrm{C}$, et al. Identification and characterisation of osteoarthritis patients with inflammation derived tissue turnover. Osteoarthr Cartil. 2014;22:44-50.

23. Skjøt-Arkil H, Schett G, Zhang C, Larsen DV, Wang Y, Zheng Q, et al. Investigation of two novel biochemical markers of inflammation, matrix metalloproteinase and cathepsin generated fragments of C-reactive protein, in patients with ankylosing spondylitis. Clin Exp Rheumatol. 2012;30:371-9.

24. Genovese MC, Fleischmann R, Kivitz AJ, Rell-Bakalarska M, Martincova R, Fiore $S$, et al. Sarilumab plus methotrexate in patients with active rheumatoid arthritis and inadequate response to methotrexate: results of a phase III study. Arthritis Rheumatol. 2015;67:1424-37.

25. Rafique A, Martin J, Blome M, Huang T, Ouyang A, Papadopoulos N Evaluation of the binding kinetics and functional bioassay activity of sarilumab and tocilizumab to the human il-6 receptor (il-6r) alpha. Ann Rheum Dis. 2013;72 Suppl 3:A797.
26. Huizinga TW, Fleischmann RM, Jasson M, Radin AR, van Adelsberg J, Fiore S, et al. Sarilumab, a fully human monoclonal antibody against IL-6Ra in patients with rheumatoid arthritis and an inadequate response to methotrexate: efficacy and safety results from the randomised SARIL-RAMOBILITY Part A trial. Ann Rheum Dis. 2014;73:1626-34.

27. Siebuhr AS, Bay-Jensen AC, Leeming DJ, Plat A, Byrjalsen I, Christiansen C, et al. Serological identification of fast progressors of structural damage with rheumatoid arthritis. Arthritis Res Ther. 2013;15:R86.

28. Okada $Y$, Nagase $H$, Harris Jr ED. A metalloproteinase from human rheumatoid synovial fibroblasts that digests connective tissue matrix components. Purification and characterization. J Biol Chem. 1986;261:14245-55.

29. Garnero P, Thompson E, Woodworth T, Smolen JS. Rapid and sustained improvement in bone and cartilage turnover markers with the antiinterleukin-6 receptor inhibitor tocilizumab plus methotrexate in rheumatoid arthritis patients with an inadequate response to methotrexate: results from a substudy of the multicenter double-blind, placebo-controlled trial of tocilizumab in inadequate responders to methotrexate alone. Arthritis Rheum. 2010;62:33-43.

30. Fadda S, Hamdy A, Albulkhair E, Elsify HM, Mostafa A. Serum levels of osteoprotegerin and RANKL in patients with rheumatoid arthritis and their relation to bone mineral density and disease activity. Egypt Rheumatologist. 2015;37:1-6.

31. Oelzner $P$, Franke $S$, Lehmann $G$, Eidner $T$, Müller A, Wolf $G$, et al. Soluble receptor activator of NFkappa B-ligand and osteoprotegerin in rheumatoid arthritis - relationship with bone mineral density, disease activity and bone turnover. Clin Rheumatol. 2007;26:2127-35.

32. Torring $\mathrm{O}$. Effects of denosumab on bone density, mass and strength in women with postmenopausal osteoporosis. Ther Adv Musculoskelet Dis. 2015;7:88-102.

33. González-Alvaro I, Ortiz AM, Tomero EG, Balsa A, Orte J, Laffon A, et al. Baseline serum RANKL levels may serve to predict remission in rheumatoid arthritis patients treated with TNF antagonists. Ann Rheum Dis. 2007;66:1675-8

34. Simonet WS, Lacey DL, Dunstan CR, Kelley M, Chang MS, Lüthy R, et al. Osteoprotegerin: a novel secreted protein involved in the regulation of bone density. Cell. 1997:89:309-19.

35. Kanbe K, Nakamura A, Inoue Y, Hobo K. Osteoprotegerin expression in bone marrow by treatment with tocilizumab in rheumatoid arthritis. Rheumatol Int. 2012;32:2669-74

36. van Tuyl LH, Voskuyl AE, Boers M, Geusens P, Landewé RB, Dijkmans BA, et al. Baseline RANKL:OPG ratio and markers of bone and cartilage degradation predict annual radiological progression over 11 years in rheumatoid arthritis Ann Rheum Dis. 2010;69:1623-8.

37. Terpos E, Fragiadaki K, Konsta M, Bratengeier C, Papatheodorou A, Sfikakis PP. Early effects of IL-6 receptor inhibition on bone homeostasis: a pilot study in women with rheumatoid arthritis. Clin Exp Rheumatol. 2011;29:921-5

38. Garnero P, Borel O, Byrjalsen I, Ferreras M, Drake FH, McQueney MS, et al. The collagenolytic activity of cathepsin $\mathrm{K}$ is unique among mammalian proteinases. J Biol Chem. 1998;273:32347-52.

39. Makrygiannakis D, af Klint E, Catrina SB, Botusan IR, Klareskog E, Klareskog L. Intraarticular corticosteroids decrease synovial RANKL expression in inflammatory arthritis. Arthritis Rheum. 2006:54:1463-72

40. Karsdal MA, Woodworth T, Henriksen K, Maksymowych WP, Genant H, Vergnaud $P$, et al. Biochemical markers of ongoing joint damage in rheumatoid arthritis-current and future applications, limitations and opportunities. Arthritis Res Ther. 2011;13:215. 